\title{
Problems and Progress in Photography*
}

\author{
By OlaF Bloch, Research Laboratory, Ilford, Ltd.
}

$\mathrm{P}$ HOTOGRAPHY is the recording angel of the arts and sciences, but it is to be hoped that angels of other classifications do not have to endure the insistent requests for materials possessing incompatible properties which are demanded of the photographic chemist. Perhaps the most difficult problems are presented by the physicists and astronomers, who require grainless emulsions of extremely high speed, sensitive to all the visible spectrum, or any portion of it-with extensions into the ultra-violet and infra-red regions !

For work in the neighbourhood of the $\mathrm{H} \alpha$ line, two types of emulsion are now available, one which attempts to give the astronomers a maximum sensitivity at $\lambda 6500$, and another, which has fairly even sensitivity round this region of the spectrum, extends well beyond. The spectrum of meteors has been successfully photographed by Williams, of the Steward Observatory, and for this purpose an emulsion of great speed and high green sensitivity was necessary. Other recent emulsions include one with a maximum at $\lambda 5300$ for work on the solar corona, and another, for lunar disc photography, of fine grain and high contrast, where an attempt has been made to compensate for the low speed by extreme colour sensitising.

In the region of atomic physics, properties of another kind are desirable. For $\alpha$-particle and proton track work, it is essential that the grain of the emulsion should be fine, resolving power high, and that the unexposed portions should yield but few or no silver grains upon development. Normally, emulsions have about $3.5 \times 10^{6}$ such grains per $\mathrm{cm} .{ }^{2}$ when developed without exposure, the average size of these being dependent upon the size of the silver halide crystals; but a recent product of the emulsion maker's art gives almost complete freedom from these.

Quite different requirements are necessary for work with atomic rays and in the extreme ultraviolet. Here the radiation has little penetrating power and is readily absorbed by gelatine. Hence, only sufficient gelatine must be used to bind the silver halide, or the crystals of the latter must be made to project beyond the gelatine surface-a rook-garden effect! The Schumann plates, always somewhat untrustworthy, are of the former type, and recently an emulsion of the latter kind has been produced which appears to be sturdier and more constant in its properties.

There are two main sections in photography:

* Substance of three lectures delivered at the Royal Institution on Oetober 30 , November 6 and 13,1934 . first, its applications, including the important function of recording; and secondly, the internal problems of the subject, dealing largely with the mechanism of emulsion making and the effect of light upon the sensitised silver halide. The function of gelatine in emulsion making is complex : first, it holds silver halide in suspension, a suspension by no means perfect, since sedimentation usually takes place when the liquid emulsion is allowed to stand. Secondly, it is the principal means by which speed can be obtained; and thirdly, it prevents too rapid reduction of the unexposed silver halide in the developer, whilst permitting a sufficiently slow reduction of the latent image to enable control to be exercised. The amount of gelatine necessary to produce suspension is very small, so little as $1 \mathrm{gm}$. of gelatine per $100 \mathrm{gm}$. of silver halide being effective. Discussion is still rife as to the mechanism of the silver halide gelatine system, one argument being that bodies in the gelatine, having the iminazole and azine linkages, tend to form adsorbed layers of the protective colloid on the silver halide surfaces. It has not been found possible to remove the gelatine entirely from the latter by centrifuging and subsequent washing, and whether the retention of the gelatine is mechanical or otherwise, it would probably not be without effect upon the resulting emulsion.

The silver halides are all light-sensitive and darken when exposed to light, free halogen and metallic silver being amongst the reaction products when the intensity is sufficiently high, but exceedingly low intensities suffice to produce a developable latent image when the emulsion is in a highly sensitive condition. Very little is known about the changes which occur in these circumstances. It is at present generally held that the first action of light is the splitting off of electrons from the bromine ions in the silver bromide, with the consequent discharge of silver ions forming atoms of free halogen and free silver, the emulsion possibly becoming developable by reason of the altered position of the electric charges. The photoelectric effect does not appear to play a part, but it has been found that the photo-conductance of silver bromide in exceedingly thin layers increases and decreases in a manner corresponding to its spectral sensitivity in energy terms, the photosensitive units probably being the bromide ions. Also, in the case of a photo-voltaic cell consisting of electrodes of silver bromide coated on silver and in a bromide solution, the illumination of one pole sets up potential differences. In most of this 
work the suggestions are purely theoretical, or have resulted from experiments on precipitated or deposited silver bromide per se, or of silver bromide crystals in an isolated condition. Little of the work deals with the silver halide in its actual emulsion form. Such evidence as can be obtained depends almost entirely upon the action of a reducer in order to study the effect of light in producing the latent image, and we have very little real knowledge of the state of affairs existing in the silver halide crystal in emulsion form either before or after exposure. The conclusions reached at present are largely inferential and this remains one of the unsolved problems of photography. Also, we have no exact knowledge as to the relationship between light quanta and latent image. acts by deforming the ultimate structure of the silver bromide, thus affecting the silver and bromine ions, so that the photo-chemical decomposition might be more localised with a more ready splitting off of the bromine electrons. There is no doubt of the main facts, but it seems probable that it is only a particular case, since other substances not containing sulphur can act similarly, though less effectively, in the case of the desensitised gelatines.

A great deal has been done in the investigation of the phenomenon of development, and, in general, two views may be said to hold the field: one is that the reduced silver is dissolved in the developer and re-deposited; the other formulates the idea that the reducing

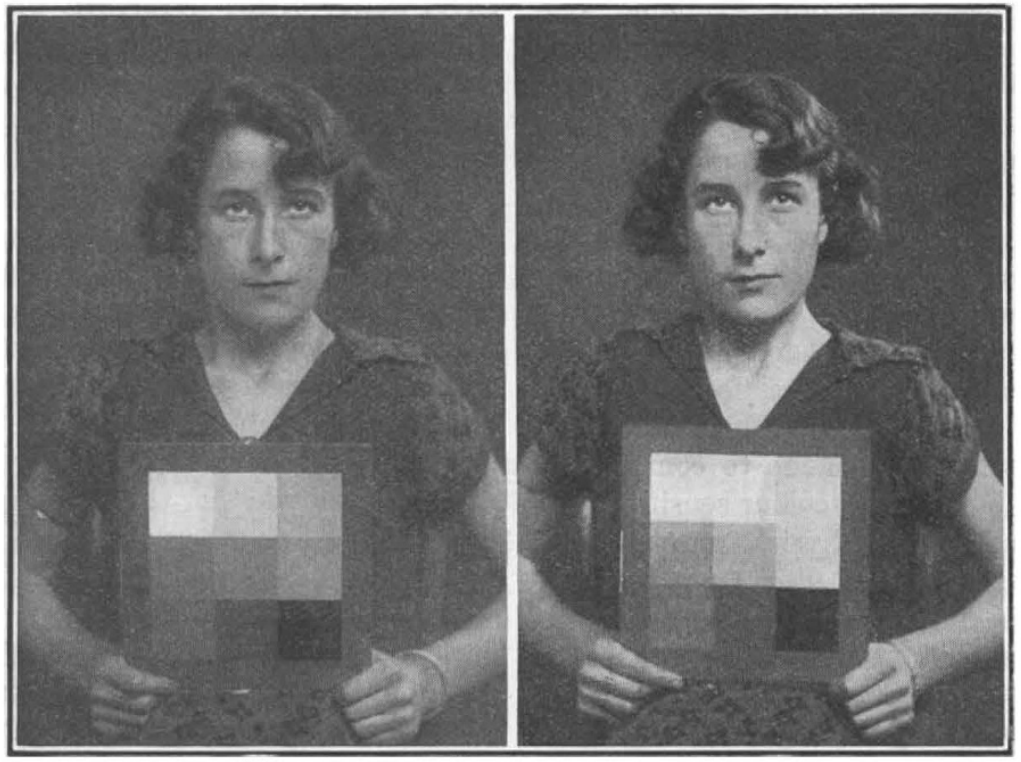

(a)

(b)

FiG. 1. (a) Negative made on the unexposed portion of the characteristic curve of an emulsion and developed for a long time to secure maximum contrast. (b) Negative made on the straight line portion of the characteristic curve of the same emulsion and developed for a short time to sccure minimum contrast.

One of the interesting discoveries was the existence of minute quantities of sulphur compounds of the thiocarbamide type in gelatine. When the gelatine was sufficiently purified to remove these, emulsions prepared with it were of low sensitivity, and the restoration of the sulphur-containing compound, at almost any stage of the emulsion making process, restored the sensitivity. In practice, the original sensitivity cannot be greatly exceeded by increasing additions of the sulphur compound beyond a certain point, since fog results. There would appear to be an adsorption of the sulphur compound on to the silver halide, subsequent reaction yielding silver sulphide in the form of specks which are supposed to be the sensitive nuclei.

Much work has been done upon this subject, and it has been supposed that the silver sulphide ions of the developer are first adsorbed on to the silver, a re-arrangement afterwards occurring which gives metallic silver and oxidised reducer. The function of gelatine as a protective agent in reduction is not complete since all emulsions show some reduction if the reducer is allowed to act for a sufficiently long period.

The mode of expression of the speed of an emulsion is still a vexed question, since it is impossible that one figure can express a variety of different properties in any one substance. Speed may depend upon the portion of the emulsion curve which is employed for the particular purpose in hand (Fig. 1, $a$ and $b$ ), and a knowledge of speed alone need by no means be the first considerationgradation, resolving power, graininess and other properties may be of equal or greater importance. 
The older Hurter and Driffield method of expressing the speed depends upon the straight line portion of the characteristic curve, and this is a measure of the contrast (gamma) of the emulsion as well as of its speed, and is often inadequate. More modern methods, including the German DIN (das ist normal) system, depend upon some function of the under-exposure curve and yield a speed number which is, perhaps, more in accord with the requirements of those who take instantaneous camera pictures. There are many obstacles in the way of giving a brief statement as to the properties of any one photographic emulsion, the variation in the gradation and length of both the underexposure and the straight line portion of the curve, the shape of the over-exposure curve and the exposure point at which it begins to function, the failure of the emulsion to obey the reciprocity

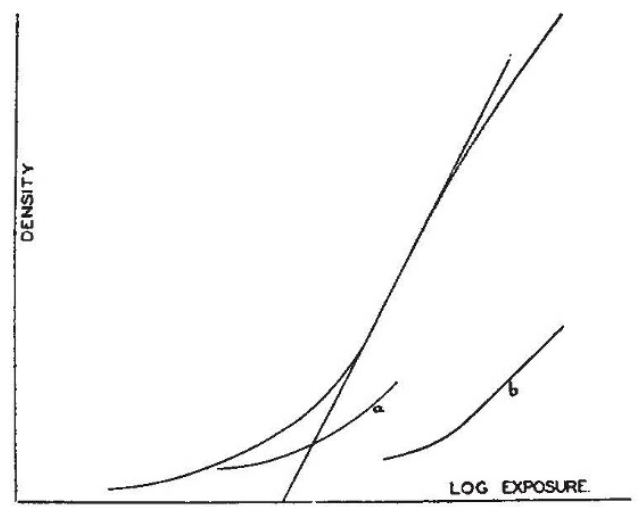

FIG. 2. Characteristic curve of the emulsion used in Fig. 1. Portion used in Fig. $1 a$ is marked $a$; that used in Fig. $1 b$ is marked $b$.

law, may all enter into one or other of the uses to which the sensitive material is to be put. It is certainly important that some indication of the photographic quality of the material used should be given, but it is not possible to accomplish this satisfactorily until a much larger proportion of the camera-using public is educated to a higher photographic standard.

Two important properties of emulsions are resolving power and graininess. The former is a very complex quality, depending upon a large number of factors. It has been defined as the capacity of an emulsion to resolve a number of lines and spaces per millimetre, the lines and spaces being of equal dimensions. Some of the factors influencing it are the maximum contrast obtainable and the shape of the characteristic curve, the amount of scatter due to the film, its graininess, its spectral sensitivity, the size of the image, etc. Other factors are shape, range of contrast and spectral quality of the object, the amount of exposure, the treatment in processing, the aperture of the lens and the spectral quality of the light used for taking the photograph. Some of these operate in antagonistic directions: for example, resolving power decreases with grain size, but since emulsions of very small grain size are somewhat slow, their use is impracticable where high speed is required. In the same way, small lens apertures are not possible in the same circumstances.

It is necessary, for many purposes, to enlarge the photographic image; for this reason, the cinematograph and the increasing use of the small precision camera have made the question of graininess an important one. Graininess might be defined as the distance at which the image of the silver deposit no longer appears uniform at constant magnification, or the magnification at which the silver deposit no longer appears uniform viewed from a constant distance. The question is a physiological one and a standard is therefore difficult to set up. Various methods, mostly visual, and chiefly comparative in character, are used for determining this property. Graininess depends mainly upon the emulsion itself, factors being the size of the original silver halide crystals and the extent to which clumping occurs, either in the emulsion itself, or as a result of development. Modern emulsion making has shown considerable advances in the direction of producing faster emulsions possessing a relatively fine grain, and it seems probable that these methods can be still further developed.

Optical sensitising is the term applied to the alteration of the spectral sensitivity of an emulsion by the addition of a dyestuff which is adsorbed by the silver halide and confers upon it an added sensitivity in the neighbourhood of the absorption of the dyestuff. So far as experimental data are at present available, the dye can be removed by the use of an inert solvent suitable for the particular dyestuff, with consequent loss of the added sensitivity. The sensitising power varies with the chemical composition of the dye, and the addition of substituent groupings can have a large effect, or the substitution of one grouping for another (for example, the change from a dimethyl to a diethyl grouping) can cause marked deterioration or improvement.

Ordinary emulsions are sensitive from about $\lambda 2100$ to about $\lambda 5200$, and optical sensitising does not greatly affect this inherent sensitivity. The amount of dye needed for optimal sensitising can be extremely small; in the case of one dye, for example, it is of the order of $1 \mathrm{gm}$. molecule of the dyestuff to $357,000 \mathrm{gm}$. molecules of silver bromide. In another case, at the other end of the scale, it is as large as $1 \mathrm{gm}$. molecule of the dyestuff to $6000 \mathrm{gm}$. molecules of silver bromide. 
The smaller grains of the emulsion adsorb more dye than the larger, possibly owing to their greater area per unit mass, and this accounts for the higher contrast obtained when exposure is made to light of a longer wave-length than that to which the emulsion was originally sensitive, since the smaller grains give steeper gradation than the larger and become more highly sensitive in consequence of the increased dye absorption.

Bancroft, Sheppard and many others have put forward a number of theories to account for the phenomenon of colour sensitising, and we have still almost everything to learn about its mechanism, but a good deal of recent work is likely to go far to clear up the chemistry of the dyestuffs employed. We can classify these into a number of chemical groups, and can generally say which particular compounds are likely to sensitise and which are not, from a consideration of their constitution; we can forecast with fair accuracy the region over which they are going to sensitise the emulsion, and in some cases foretell whether they will be weak or powerful sensitisers. In the older days, erythrosine was almost the only dye used, and sensitised the emulsion up to about $\lambda 5800$, but with the advent of the cyanine group of dyestuffs, great progress has been made and it is now possible to sensitise an emulsion, at will, to almost any part of the visible spectrum. This class may be thus represented:
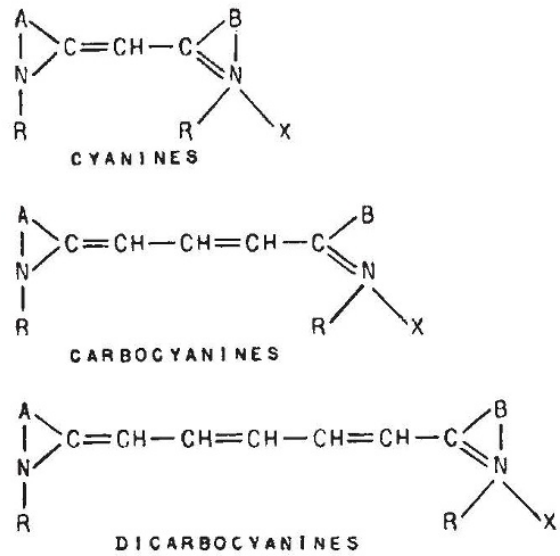<smiles>[R]B1C2(C=CC=CC=CC3=CN3[R])BN([Y])C12[Y]</smiles>

$A$ and $B$ represent several types of nuclei and the compound may be unsymmetrical when $A$ and $B$ are different nuclei. The number of $\mathrm{CH}$ groups is odd. $R$ may be methyl, ethyl, allyl, etc., and $X$ may be chloride, bromide, iodide, $p$-toluene sulphonate, etc. Substitution may also take place in the chain, where one of the CH groups may be replaced by an alkyl, aryl, ethylene or phenylene group, or there may even be a halogen substituent in the chain. Generally speaking, as one increases the chain length, the dye becomes more unstable and the photographic products prepared with it more unstable. As the length of the chain is increased, the dye absorption and sensitising power travel towards the red end of the spectrum, each additional pair of $\mathrm{CH}$ groups moving the absorption by about $1100 \mathrm{~A}$. towards the red end of the spectrum.

The case of the three carbocyanines with the following formulæ is of interest :
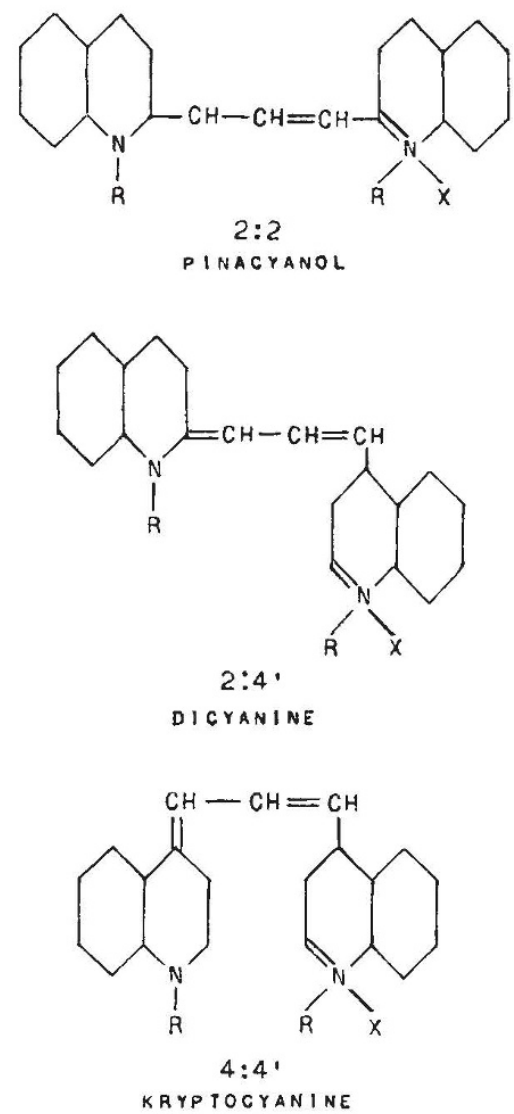

The sensitising chain is from the nitrogen in one nucleus to the nitrogen in the second nucleus. Hence, the three isomers, the $2: 2^{\prime}$, the $2: 4^{\prime}$ and the $4: 4^{\prime}$ compounds, sensitise progressively farther into the red.

Many photographic research laboratories are actively at work on the production of new sensitising dyestuffs, since the use of colour sensitive emulsions is varied and important. Competition in the photographic industry is very keen and considerable progress has been made in recent years, but in many branches empiricism is still much in advance of proven theory. 